\title{
Some results of stellar activity studies that should be known for every solar physicist (invited talk)
}

\author{
R.E.Gershberg \\ Crimean Astrophysical Observatory, Nauchny, Crimea,98409, Ukraine \\ email:gershber@crao.crimea.ua
}

\begin{abstract}
Results of detailed investigations of the whole variety of solar activity events are widely used in studies of relevant stellar activity manifestations. On the other hand, investigations of a plenty of active stars which differ in mass and age lead to determination of the decisive factor of the solar activity evolution and give ideas on the past and the future of this activity.
\end{abstract}

It is generally known that the Sun is an ordinary dwarf star of the G spectral type. Therefore one could think that a strategy to look for the solar type activity on stars should consist of looking for events analogous to the solar ones on other $\mathrm{G}$ stars. However, a real history of science is not so straightforward and trivial one.

In the end of the 1930s, during photographing of the sky, short-lived optical flares with amplitudes up to 1-2 stellar magnitudes have been registered on cool M dwarfs. In 1940s the first spectra of such flares have been obtained.

At that time it was hard to imagine that these events which lead to cardinal changes in both brightness and spectrum of a star are relevant to processes on the Sun, whose warm rays give us conditions for comfortable life. But it is the truth. The solar activity analogues should be searched for not on stars as close as possible to the Sun, but on objects where such analogues are the most noticeable. As far as optical flares are concerned they are indeed the most noticeable on dwarfs of late spectral types with faint photospheric radiation that makes flares to have the highest contrast and therefore discoverable.

Studies of many hundreds of flares indicated that they have a power-law distribution along radiation energy and the similar distribution was found for solar flares. In Fig.1 a summary plot of cumulative energy spectra of optical flares is given. It shows that the radiation energy of the strongest stellar flares exceed the solar ones by 4 orders of magnitude.

Fig.2 slows a sequence of development of spectral features of the UV Cet strong flare, and this sequence is similar to the solar one.

Up to date almost all the events known in solar flares have been discovered on stars of middle and low masses: flares in optics, at meter radio waves and in moderate X-rays, in ultraviolet and in soft X-rays, events in far ultraviolet with a duration of several days and microwave flash of minute durations with $100 \%$ circular polarization. Dark spots that create a noticeable photometric effect exist on the stars - analogue to sunspots.

Non-homogeneous chromospheres allow to estimate stellar rotational periods and longterm observations of chromospheric emission permit to detect data on activity cycles. About $1 / 3-1 / 2$ of the solar type stars show activity cycles and their durations are from 3 up to 25 years. 


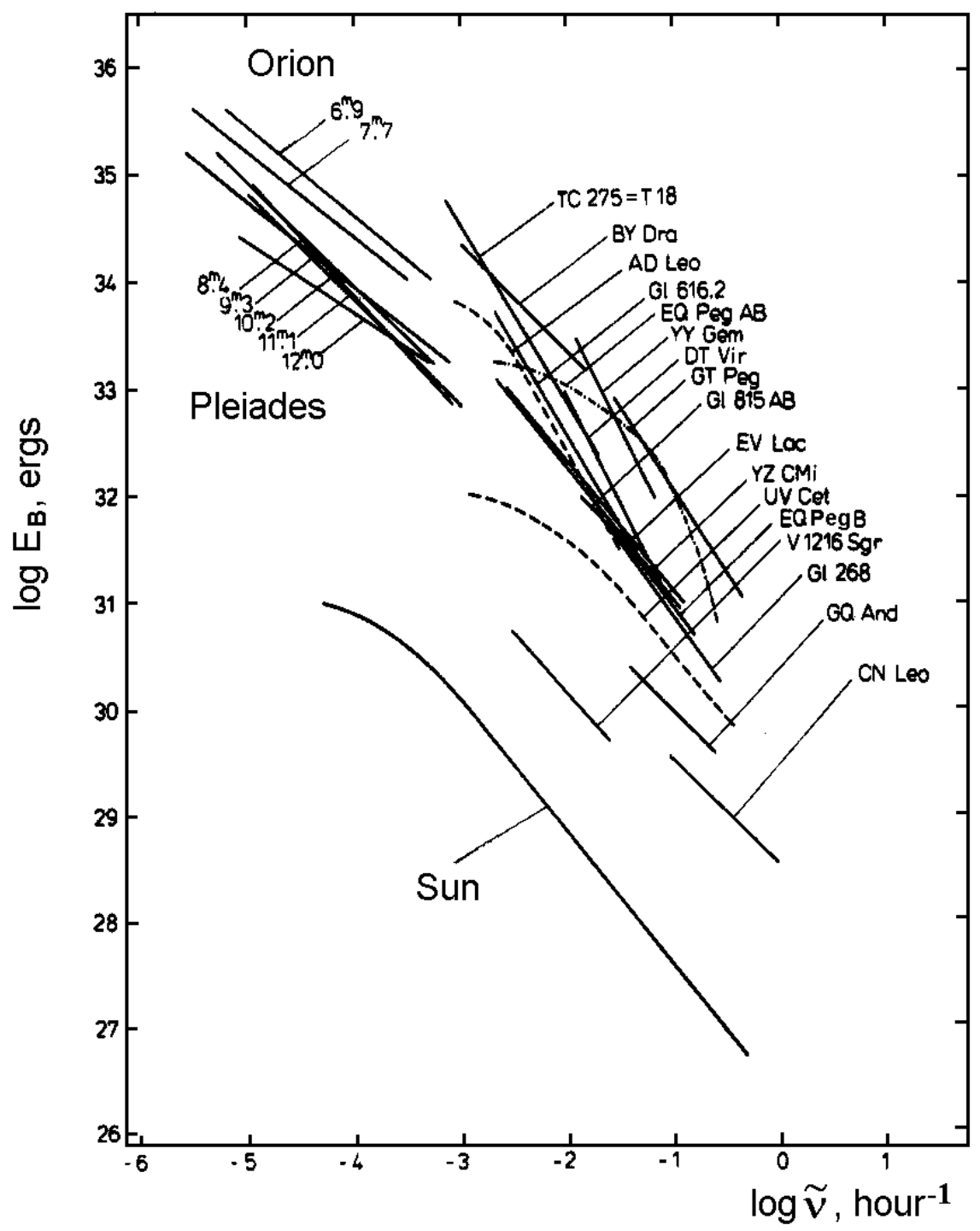

Figure 1. Flare cumulative energy spectra of stars in the solar vicinity, in Pleiades and Orion clusters, and on the Sun. (Gershberg et al., 1987).

Magnetometric observations revealed the presence of strong photospheric magnetic fields. Up to date such measurements have been carried out for 16 stars and shown strengths $\mathrm{B}=1.0-3.5 \mathrm{kGs}$ and filling factors $\mathrm{f}=0.02-0.70$.

It should be emphasized that there is not only a superficial resemblance between solar and stellar activity, but the Sun does fit into general correlations found for stars. Such an example is the characteristics of spottedness. As in the flare case, we have here an essential difference in scale: the total area of sunspots does not exceed $0.5 \%$ of the solar surface while starspot areas cover up to $30-40 \%$ on the most active stars. However, there exist certain general similarities: in lower boundaries of spot belts, in mean latitudes of starspots, in their absolute and differential temperatures relative to the photospheres see Fig.3. 


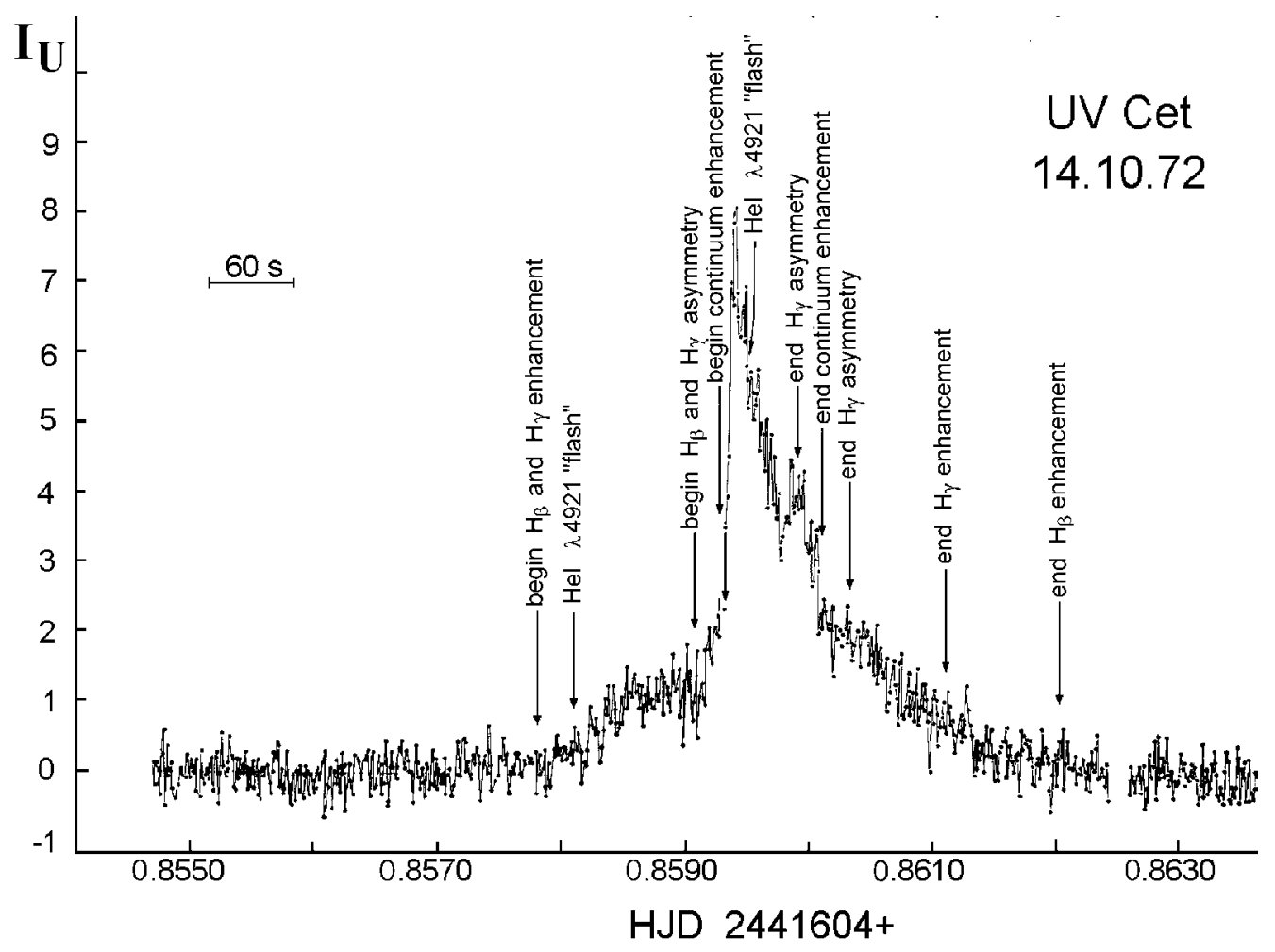

Figure 2. Light curves and temporal sequence of appearance and dispearance of different spectral features in the UV Cet flare (Bopp and Moffett, 1973).

For interpretations of stellar activity events stellar physicists use widely findings obtained in the solar physics. However, there are plenty of stars, and they differ in luminosity, mass and age. Systematic investigations of a broad range of stars using methods of contemporary multi-wavelength astrophysics allowed to discover the decisive factor of the stellar activity under consideration and to find its evolutionary trend:

The decisive factor of the solar type activity is a stellar rotation, and its evolutionary braking is a cause of the activity decay

It is obvious that this fundamental result could not be reached by solar studies only. Some evidences supporting this statement are following.

Fig. 4 gives a plot of an observed secular braking of rotations of the solar-type dwarfs.

In Fig. 5 the dependence of the ratios $L_{X} / L_{b o l}$ on stellar rotational periods for narrow ranges of $\mathrm{B}-\mathrm{V}$ is shown. The figure discovers a universe dependence $L_{X} / L_{b o l} \sim P_{\text {rot }}^{-2}$ and saturation at the level $L_{X} / L_{b o l}=10^{-3}$.

Fig.6 shows monotonous decrease of X-ray coronal radiations, of $\mathrm{C}$ IV emission from transition regions, and of $\mathrm{Mg}$ II emission of upper chromospheres and the starspot photometric effect on increasing of stellar rotational periods. The Sun also fits in these common correlations.

In Fig. 7 the dependence of the calcium emission on the Rossby number that is a ratio of a stellar rotational period to a convective turnover is given for nearby stars and Fig.8 shows an observed secular decay of the stellar calcium emissions in stellar groups of different ages. 

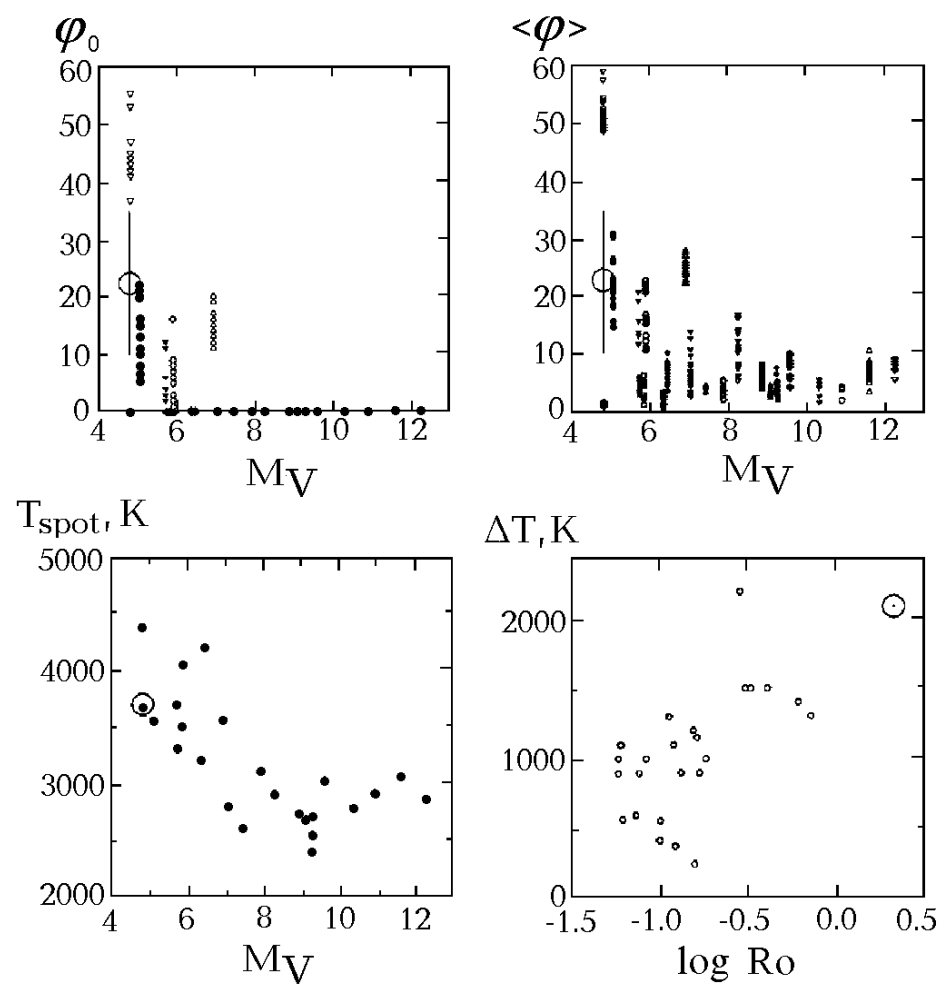

Figure 3. The confrontations of calculated parameters of starspots of red dwarfs - latitudes of lower boundaries of spot belts $\varphi_{0}$, mean latitudes of starspots $\langle\varphi\rangle$, spot temperatures and temperature differences of quiet photospheres and starspots - with the global stellar parameters - absolute stellar magnitudes $M_{V}$ and the Rossby numbers Ro (Alekseev, 2001).

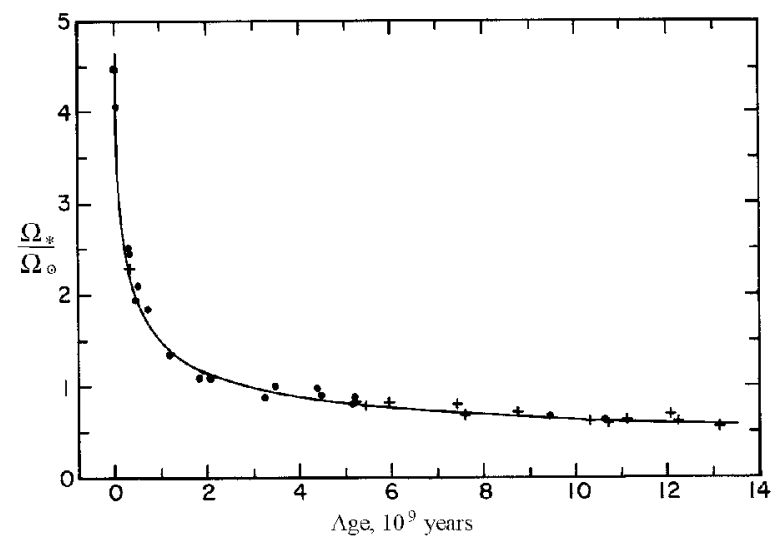

Figure 4. Angular rotational velocities for solar-type stars (Barry, 1988)

Fig.9 presents observed and calculated decreases of the coronal and the chromospheric emissions based on stellar rotation braking computations.

This set of figures allow us to estimate the activity levels of the Sun for several billion years in the past and in the future. It is important in discussing a history of the Solar system in total and of ecology of the Earth in particular. 

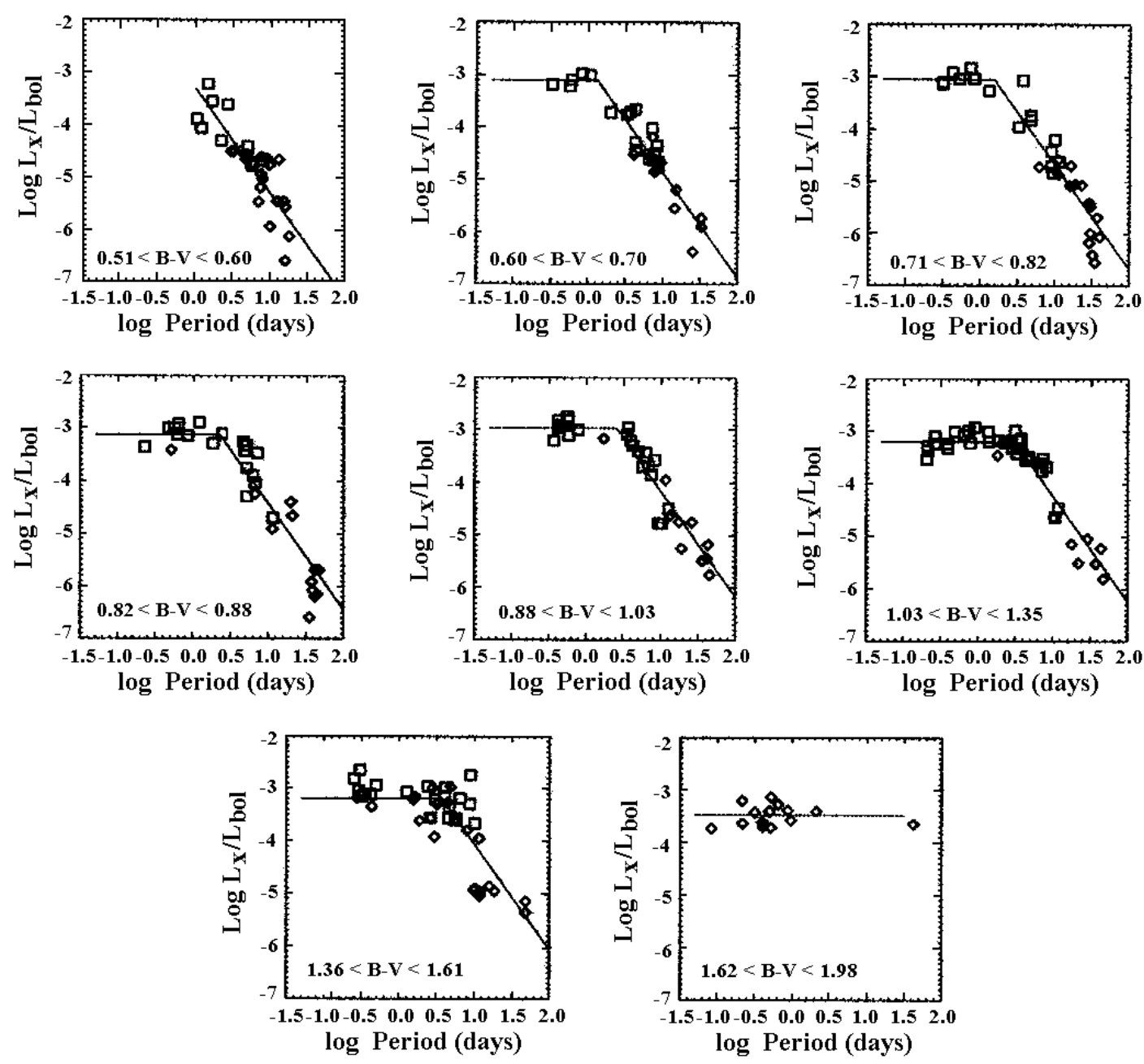

Figure 5. Dependences of the ratio $L_{X} / L_{b o l}$ on rotational periods for different B-V intervals (Pizzolato et al., 2001).

Between the stellar rotation as the ultimate energy source of the stellar activity under consideration and its observed manifestations there is the most movable physical factor - magnetic fields. Variety of structures and strengths of these fields, mechanisms of their generation and dissipation define richness of the activity events. It is important to say, that within the framework of stellar magnetic activity there are such events that do not take place on the Sun. Thus, in binary systems the braking of rotation by magnetized stellar wind does not work in practice, since a rotational momentum is fed constantly by an orbital momentum, and a high level of activity is maintained for many billion years. Then, on the most fast rotators a saturation of stellar atmospheric radiation takes place as it was shown in Fig.4, while such a saturation does not exist on the Sun. On the most active flare stars there exists permanently a very hot coronal component, but on the Sun such a component appears only during strong flares. Finally, an envelope structure of stellar global magnetic field appears only within a limited range of rotational velocity: 


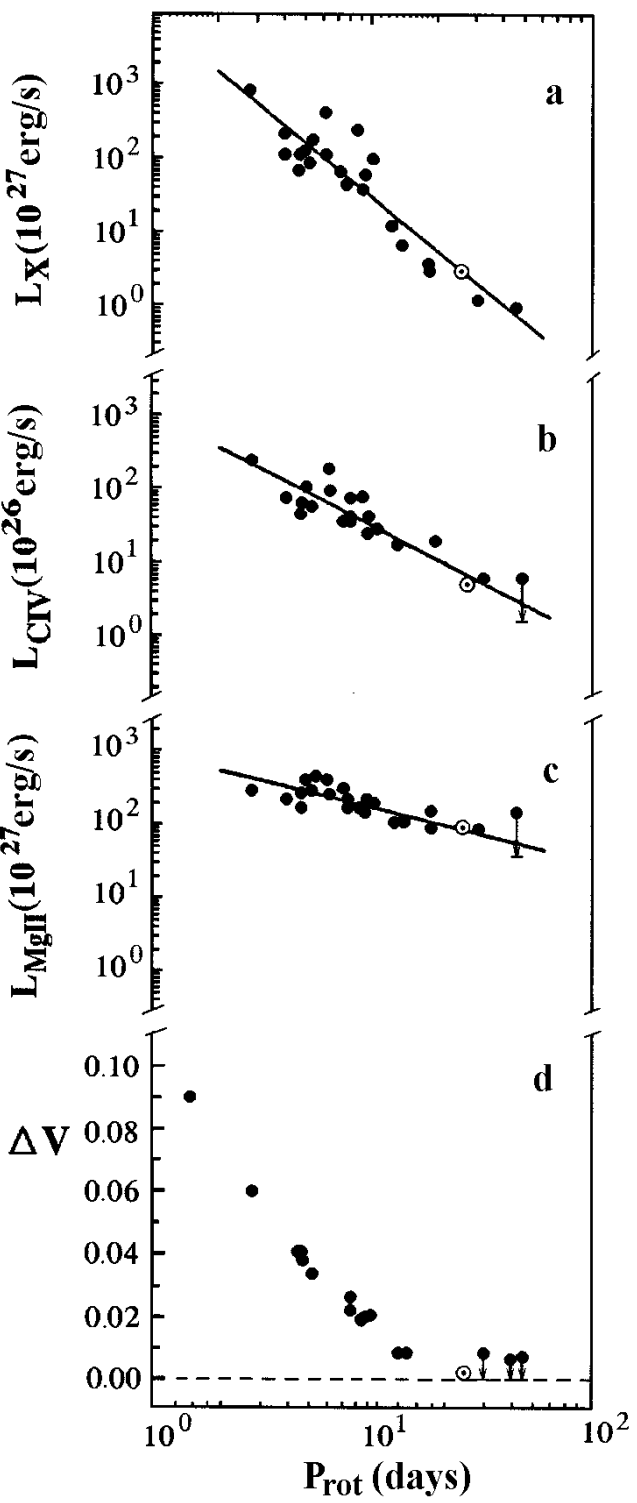

Figure 6. Decreases of various manifestations of stellar activity with increasing rotational period (Dorren et al., 1994).

on very fast and very slow rotators the global structure of stellar magnetic field differs qualitatively from the solar one.

\section{References}

Alekseev, I.Yu., 2001. Low mass spotted stars. Astroprint. Odessa.

Baliunas, S.L., Donahue, R.A., Soon, W. \& Henry, G.W. 1998, in: R.A.Donahue and J.A.Bookbinder (eds) Cool stars, stellar systems, and the Sun., ASPCS vol.154, p.153 Barry, D.C. 1988, Astrophys.J. 334, 436

Bopp, B.W. \& Moffett, T.J. 1973, Astrophys.J. 185, 239. 


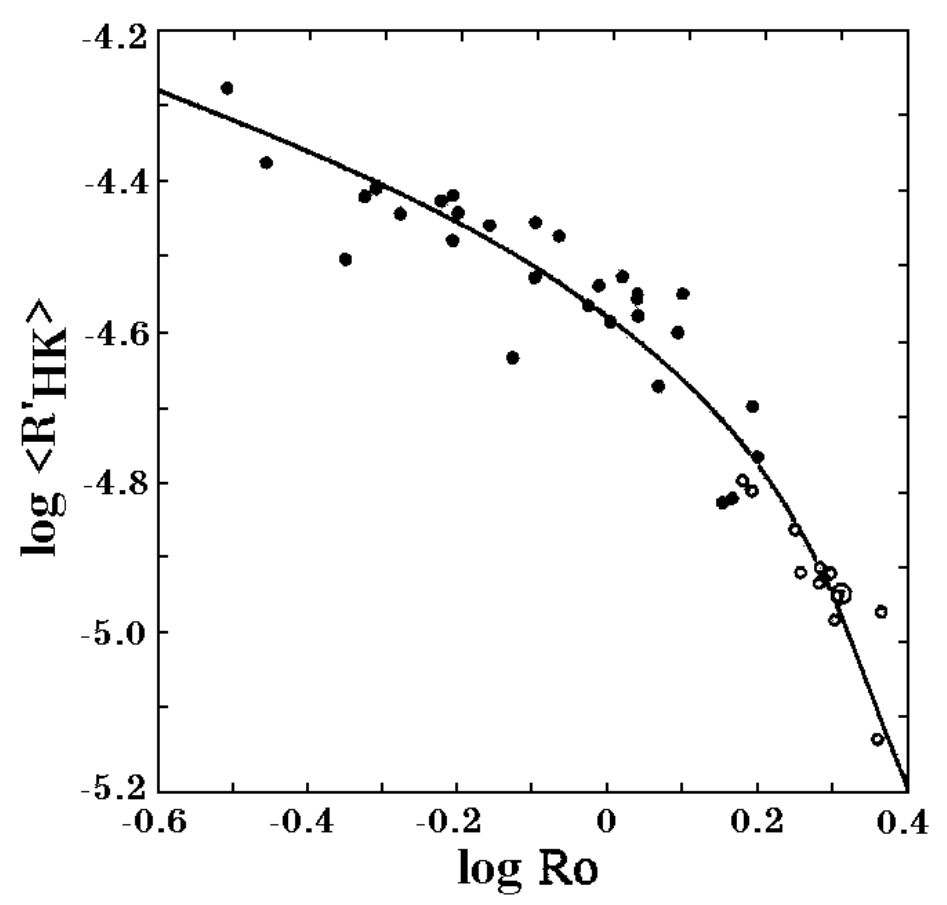

Figure 7. Dependence of the ratio $L_{C a I I} / L_{b o l}$ on the Rossby number for nearby stars (Noyes et al., 1984).

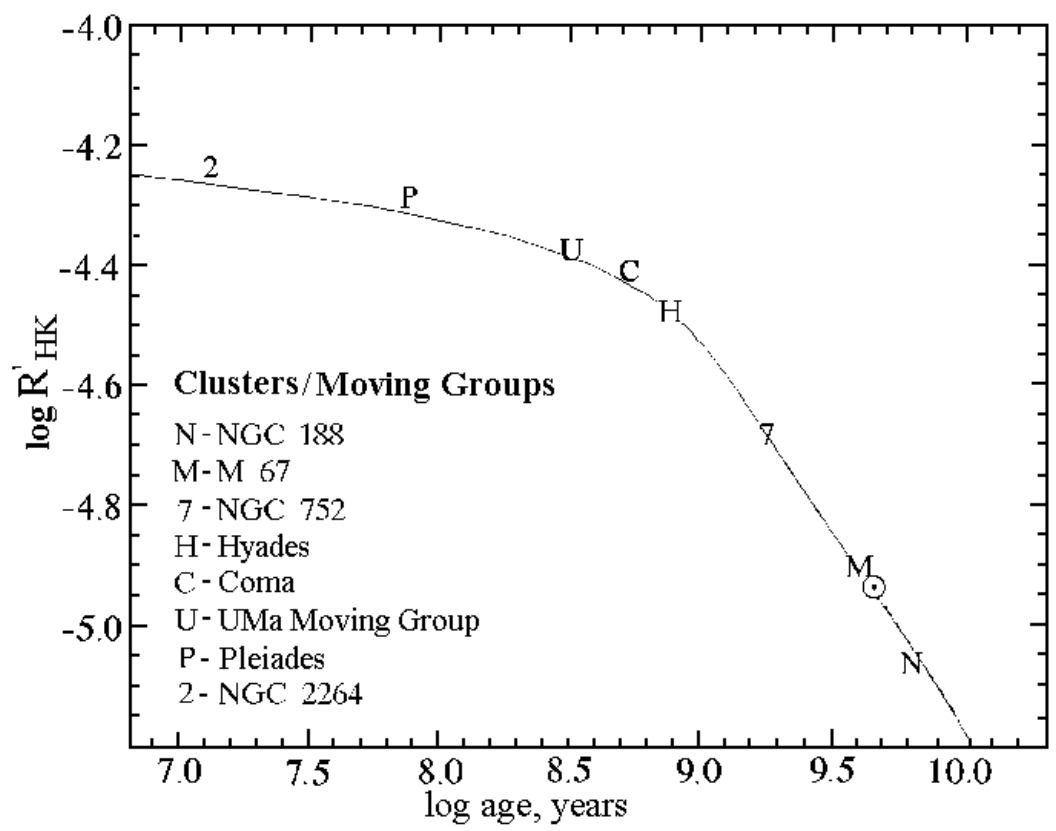

Figure 8. Dependence of the ratio $L_{C a I I} / L_{b o l}$ for solar-like stars in stellar objects of different ages (Baliunas et al., 1998). 


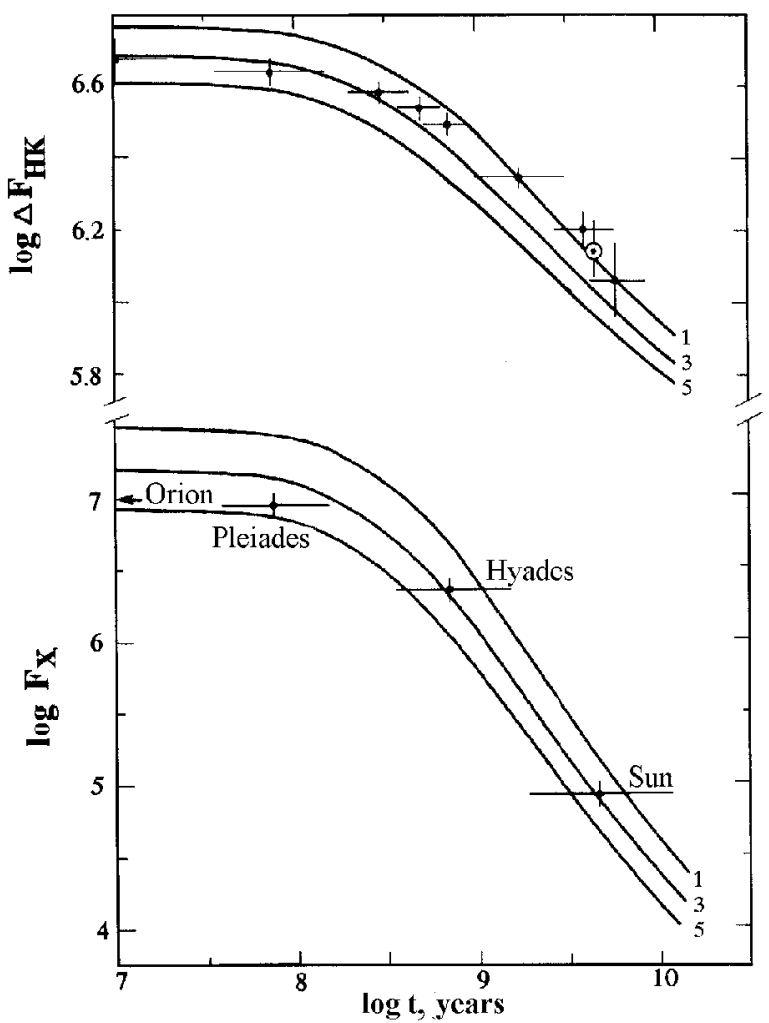

Figure 9. Observed secular decrease of Ca II and X-ray emissions and their modeling based on the stellar rotation evolution concept (Stȩpieǹ, 1989).

Dorren, J.D., Guinan, E.F. \& Dewarf, L.E. 1994, in: J.-P.Caillault (ed). Cool stars, stellar systems, and the Sun. ASPCS vol.64, p.399.

Gershberg, R.E., Mogilevsky, E.I. \& Obridko, V.N. 1987, Kinematics and physics of celestial bodies, 3, N5, 3 .

Noyes, R.W., Hartmann, L.W., Baliunas, S.L., Duncan, D.K., Vaughan, A.H. 1984, Astrophys. J., $279,763$.

Pizzolato, N., Maggio, A., Micela, G., Sciortino, S. 2002, in F.Favata and J.J.Drake (eds). Stellar coronae in the Chandra and XMM-Newton era., Proc.ESTEC Symp.ASPCS vol.277, p.557

Stẹpiè̀, K. 1989, Acta Astron., 39, 209. 